\title{
Brachyuran crabs (Crustacea: Decapoda) from the Canary Islands (eastern Atlantic): checklist, zoogeographic considerations and conservation
}

\author{
José Antonio González \\ Ecología Marina Aplicada y Pesquerías, i-UNAT, Universidad de Las Palmas de Gran Canaria, Campus Universitario de \\ Tafira, 35017 Las Palmas de Gran Canaria, Spain. E-mail: pepe.solea@ulpgc.es
}

\begin{abstract}
Summary: Just 20 years have passed since González (1995) finished one of his seminal works on decapod crustaceans of the Canary Islands, thanks to the help of the reputed carcinologists L.B. Holthuis and C.H.J.M. Fransen. This publication allowed d'Udekem d'Acoz (1999) to include the Canarian decapods in his inventory of the NE Atlantic. No checklists of decapod fauna specifically covering this area have been published since then, and an update is needed. The current list of Canarian brachyuran crabs comprises 132 species. Additional species have been recorded thanks to intensified research into deep water, natural range expansions from nearby areas, introduction by anthropogenic activities and description of new taxa; several of these changes are detailed in this review. Although the description of new brachyuran species is not expected to occur at a significant rate, an increase in the number of species from the Canaries is expected to result from trawling and dredging sampling, as well as from introduction of non-native species. For the first time, some zoogeographic comments on the Canarian brachyuran carcinofauna are made. Finally, crab species of commercial interest are listed, their current threats are identified and some updated conservation measures are proposed.
\end{abstract}

Keywords: checklist; zoogeography; conservation; Brachyura; Decapoda; Crustacea; Canary Islands; eastern Atlantic.

Cangrejos braquiuros (Crustacea: Decapoda) de las Islas Canarias (Atlántico oriental): lista comentada, consideraciones zoogeográficas y conservación

Resumen: Se cumplen 20 años desde que González (1995) publicó uno de sus trabajos seminales sobre crustáceos decápodos de Canarias, gracias a la ayuda de los reputados carcinólogos L.B. Holthuis y C.H.J.M. Fransen. Dicha publicación permitió a d'Udekem d'Acoz (1999) incluir los decápodos canarios en su inventario del Atlántico NE. Desde entonces no ha sido publicada ninguna lista completa de decápodos que cubra específicamente esta área y es necesaria una actualización. La lista actual de braquiuros canarios consta de 132 especies. Otras especies han sido citadas debido a la intensificación de las investigaciones dirigidas a aguas profundas, expansiones naturales de su rango de distribución desde áreas cercanas, introducciones causadas por actividades antropogénicas y a descripción de nuevas especies; algunos de estos cambios se detallan en esta revisión. Aunque no se espera que la descripción de nuevos braquiuros se produzca a un ritmo significativo, es esperable un incremento en el número de especies en Canarias como resultado de muestreos con arrastre bentónico y dragas y de la introducción de especies exóticas. Por primera vez, se realizan consideraciones zoogeográficas sobre la carcinofauna de braquiuros de Canarias. Por último, las especies de interés comercial son listadas, sus amenazas actuales identificadas y se proponen algunas medidas de conservación.

Palabras clave: lista; zoogeografía; conservación; Brachyura; Decapoda; Crustacea; Canarias; Atlántico oriental.

Citation/Como citar este artículo: González J.A. 2016. Brachyuran crabs (Crustacea: Decapoda) from the Canary Islands (eastern Atlantic): checklist, zoogeographic considerations and conservation. Sci. Mar. 80(1): 89-102. doi: http://dx.doi. org/10.3989/scimar.04350.10A

Editor: E. Macpherson.

Received: September 28, 2015. Accepted: November 11, 2015. Published: January 20, 2016.

Copyright: () 2016 CSIC. This is an open-access article distributed under the Creative Commons Attribution-Non Commercial Lisence (by-nc) Spain 3.0.

\section{INTRODUCTION}

Just 20 years have passed since González (1995) finished one of his seminal works on decapod crusta- ceans of the Canary Islands, thanks to the invaluable help of the reputed carcinologists L.B. Holthuis (see Holthuis 1995) and C.H.J.M. Fransen. This publication allowed d'Udekem d'Acoz (1999) to include the 
Canarian decapods in his inventory and distribution of decapods in northeastern Atlantic north of $25^{\circ} \mathrm{N}$.

The Canary archipelago is an overseas Spanish territory and an outermost European Region placed in the eastern-central Atlantic. This archipelago is situated in front of the northwestern coast of Africa, fairly close to the continents of Europe and Africa $(104 \mathrm{~km}$ from Cape Juby, Morocco) but separated from them by great depths (Fig. 1). The age of the islands varies from east to west between 19 my (Lanzarote) and 0.7 my (El Hierro). The volcanic characteristics of the Canary Islands are shown by the absence of wide insular shelves, with a bottom depth of 180-200 m near the coast. The archipelago has nearly $1300 \mathrm{~km}$ of coastline and is washed by the oligotrophic ocean (Braun and Molina 1984). The Canaries are under the influence of the subtropical gyre of the eastern-central Atlantic, which facilitates the transport of planktonic larvae and rafting organisms to the archipelago from the American, European and Northwest African coast. The average seawater temperature around the Canary Islands is $18.5^{\circ} \mathrm{C}$ in February, rising abruptly to $24^{\circ} \mathrm{C}$ in August-September (e.g. Barton et al. 1998). A mesoscale distribution of larval communities has recently been described in filaments of the upwelling system from the African coast reaching the southeast of the archipelago (e.g. Landeira et al. 2009, 2010). Also, the Canaries are geographically located on a very important maritime route, and both ships and oil platforms have been recognized as major vectors for the introduction of non-native species (e.g. González et al. 2012a, Triay-Portella et al. 2015).

Geomorphological, geographical and oceanographic particularities of the Canaries could explain the great diversity in the biogeographic patterns of the biota inhabiting this area. These physical and biodiversity characteristics, together with the climatic condition of the Canary Islands - a temperate-subtropical area-compared with the surrounding region highlight the uniqueness of the Canary Islands and their oceanographic connectivity to the adjacent waters (e.g. González et al. 2012a).

The sustainable use of marine resources and their conservation measures is a major goal on national and international policy agendas (e.g. Spalding et al. 2007). According to the comprehensive biogeographic system for classifying the oceans (for both coastal and shelf areas) proposed by Spalding et al. (2007), the Canary Islands are included in the Azores-Madeira-Canaries ecoregion (i.e. the Macaronesian archipelagos), within the Lusitanian province of the Temperate Northern Atlantic realm.

After the work of González (1995) and the compilation by d'Udekem d'Acoz (1999), Fransen and Wirtz (1997) published on Canarian decapod crustaceans, and González et al. (1996, 2000) and Quiles et al. (2002) listed crab families in the Canary Islands. Moro et al. (2014) published a list of decapods from the Canary Islands based on both material examined and in situ sightings, illustrated with colour photographs. Moreover, several authors have published updated lists of brachyuran decapod fauna at different geographical scales (e.g. Türkay 2001, Ng et al. 2008, Marco-Herrero et al. 2015). However, none of these has specifically covered the diversity found around the Canary Islands; and an update is needed for this area.

The systematic research landscape on decapod crustaceans has changed drastically in the last few decades. A great number of changes concerning the brachyuran crustacean species found around the $\mathrm{Ca}$ nary Islands have also taken place. These changes are due to systematic modifications, non-confirmed presence or newly recorded species for the area. Today's most widely used classifications have all appeared after the work of Zariquiey Álvarez (1968), and there is a concerted effort by carcinologists worldwide to check the validity of taxa using multiple tools such as ecology, larvae and genetics (Marco-Herrero et al. 2015).

The present work summarizes all changes in Canarian brachyuran carcinofauna since González (1995), provides scientists with an updated classification list, and makes for the first time a zoogeographic analysis of this important component of the marine biota of the Canary Islands. Moreover, crab species of commercial interest are listed, their current threats are identified, and some updated conservation measures are proposed.

\section{MATERIALS AND METHODS}

For the compilation of this list, all publications since 1995 about the distribution of brachyuran crabs were checked, including previous lists for the Canary Islands region (González 1995, González and Quiles 2003), data from Internet databases such as WoRMS (http://www.marinespecies.org/) and GBIF (http:// www.gbif.org/species), systematic data, new records, and unpublished or in-preparation data. Several contributions need to be highlighted here, particularly the impressive studies on European decapods (d'Udekem d'Acoz 1999, Türkay 2001), Iberian decapods (gathering many records from the Canaries, Zariquiey Alvarez 1968), and West African brachyuran crabs (Manning and Holthuis 1981), but also several specific works on brachyurans (Neumann 1996, Fransen and Wirtz 1997, Moro et al. 2014), particularly the accounts on Canarian species of several crab families (González et al. 1996, 2000, Quiles et al. 2002). Several studies on feeding habits of fishes in waters of the Canaries (Fanlo et al. 1993, 1996, Tuset et al. 1996, Dürr and González 2001, Moreno-López et al. 2002) have also been used as a source of brachyurans occurring in the area.

Some projects (DGXIV/C/1 94/034, CAMARON, PESCPROF 1-3, REDECA, AQUACRU, MARPROF) have provided us with deep-water crabs from the Canaries, collected with a panoply of fishing gear. Finally, many vouchers from the 'Museo de Ciencias Naturales de Tenerife (TFMC)', 'Museu d'Historia Natural do Funchal (MMF)', and the study collection of the 'Instituto Canario de Ciencias Marinas' (ICCM, now transferred to the 'Universidad de Las Palmas de Gran Canaria') were checked.

This checklist covers all marine brachyuran species present in the Canary Islands from the intertidal to deep water. As in previous regional studies on decapod crus- 


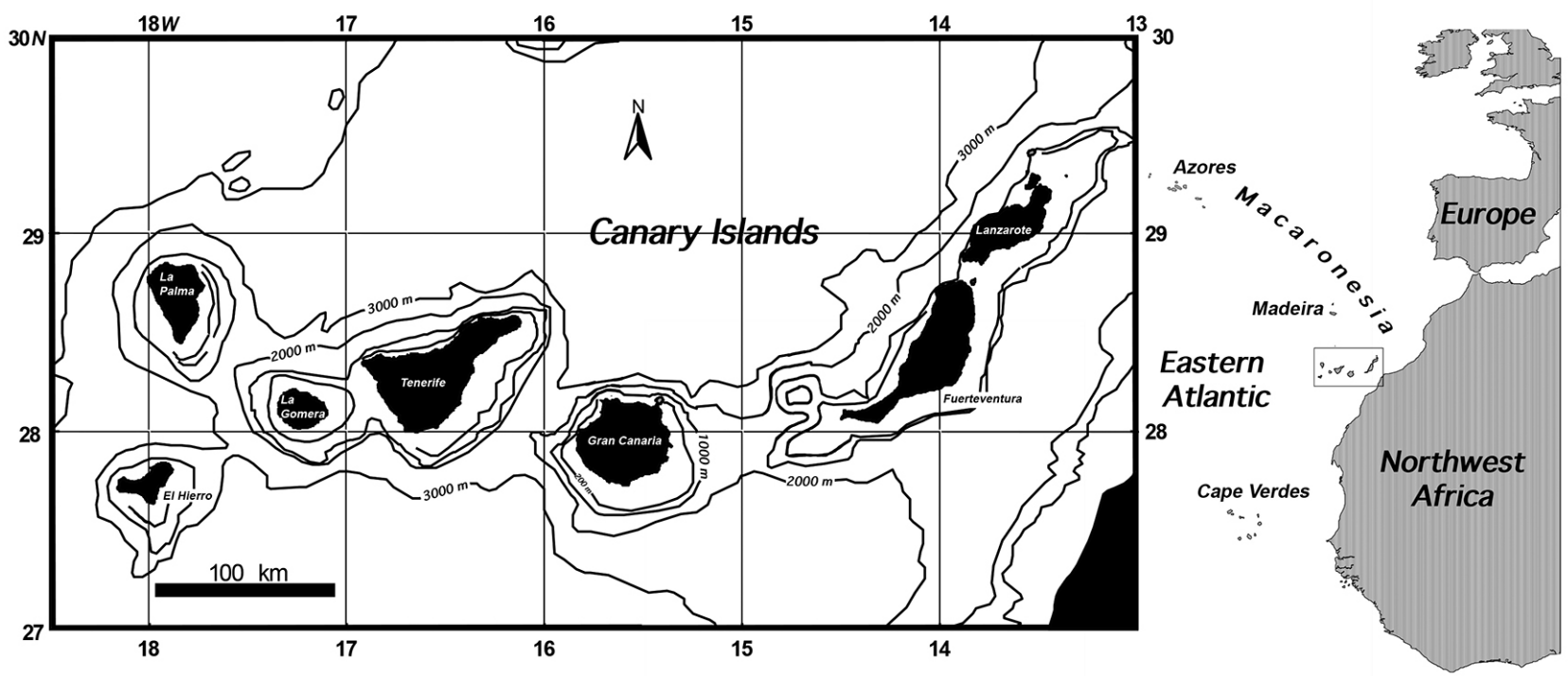

Fig. 1. - Map of the Canary Islands (northeastern Atlantic) showing their geographic situation and bathymetric characteristics.

taceans (González 1995) or fishes (Brito et al. 2002), the study area is bounded by the $30^{\circ} \mathrm{N}$ and $27^{\circ} \mathrm{N}$ parallels, the $19^{\circ} \mathrm{W}$ meridian and, in the Canaries-Africa channel, the $13^{\circ} \mathrm{W}$ meridian. The depth is generally less than $1500 \mathrm{~m}$. This area occupies a band of about $600 \mathrm{~km}$ from east to west and about $330 \mathrm{~km}$ from north to south. In the north and west the depth is greater than $4000 \mathrm{~m}$ and on the southern edge it is greater than 3500 m (Fig. 1).

This updated systematic classification follows $\mathrm{Ng}$ et al. (2008), but also takes into account the latest changes in particular taxa (e.g. Marco-Herrero et al. (2013) for Majoidea; Schubart and Reuschel (2009) and Spiridonov et al. (2014) for Cancroidea and Portunoidea). Superfamilies are listed by systematic order following the Sections and Subsections as currently accepted, and by alphabetical order within them. Families, subfamilies, genera, species and subspecies are also listed by alphabetical order within their respective superfamilies.

All changes with respect to the catalogue by González (1995) and the subsequent checklist by González and Quiles (2003) are explained, including systematic modifications, misidentifications, synonyms, species that reach Canarian waters by increasing their distribution range, invasive and introduced species in the Canary Islands, and new records from the Canaries.

Each crab species was classified as pelagic or benthic according to the main spatial distribution of their adults. Moreover, according to depth boundaries found around the Canary Islands (closely linked to regional oceanographic conditions determined by the water masses present) (Pajuelo et al. 2015), each benthic species was then assigned to a depth boundary where the species mainly occur in waters of the Canary Islands: shelf and transition area between shelf and slope (ST, 0-300 m), upper slope (US, 301-800 m), middle slope (MS, 801-2000 m) and lower slope (LS, >2000 m).

Each crab species was also assigned to a biogeographic pattern category adapted from Brito et al. (2002) and subsequent studies (e.g. Espino et al. 2014).
Recent biogeographic studies that clearly separate the coastal and shelf biota occurring in the Macaronesian ecoregion (i.e. the Azores, Madeira and the Canaries) from that occurring in the Cape Verde Islands (officially Cabo Verde) (Brito et al. 2007, Spalding et al. 2007, Brito 2010, Wirtz et al. 2013) were also taken into account. Canarian brachyuran crabs were classified into the following 11 groups according to their distribution range and the above-mentioned considerations: 1, cosmopolitan or worldwide species (COSM); 2, pantropical or circumtropical species (PANT); 3, amphi-Atlantic species of wide distribution (AAWD); 4, amphi-Atlantic species of warm affinity (AAWA); 5, eastern Atlantic species of wide distribution (EAWD); 6, eastern Atlantic cold-temperate species (EACT); 7, eastern Atlantic warm-temperate species (EAWT); 8, Atlanto-Mediterranean species (ATLM); 9, Guinean species (restricted to tropical and subtropical eastern Atlantic) (TSEA); 10, eastern-central Atlantic island species (from the Azores to Cape Verde Islands, and southwards even to St. Helena) (ECAI); and 11, Macaronesian species (around the Azores, Madeira, Savage, and/or the Canary Islands) (MAC). Two different zoogeographic approaches (consisting in describing the different components of the brachyuran fauna) were performed. A first description included all brachyuran species. For the second approach, both pelagic and deep-water benthic species (those living deeper than the transition area between shelf and slope, $>300 \mathrm{~m}$ depth) were excluded from the analysis. It is widely accepted that the set of littoral and upper-bathyal species (in the Canaries, those living shallower than the upper slope, 0-300 $\mathrm{m}$ ) better characterize the brachyuran carcinofauna of the Canary Islands and their zoogeographic affinities.

The species' occurrence around the neighbouring archipelago of Madeira (to the north) and the Cape Verde Islands (to the south) has also been recorded. Apart from publications containing well-documented first records, the main references used to check the occurrence of the brachyuran species were Manning 
and Holthuis (1981), Türkay (1982), Fransen (1991), d'Udekem d'Acoz (1999), Wirtz and d'Udekem d'Acoz (2001), Araújo and Calado (2003), González et al. (2004), and Araújo et al. $(2009,2014)$ among others.

\section{RESULTS}

A total of 132 marine crab species are reported herein around the Canary Islands (including three species of doubtful presence and another three of probable occurrence). Their spatial distribution, depth boundary, bathymetric range and biogeographic pattern are presented in Table 1. This represents a number of species close to the 140 brachyuran species reported around the Iberian Peninsula (Marco-Herrero et al. 2015), and nearly half of the 284 brachyuran species known in European waters (including the Azores, Madeira and the Canaries), with 40 freshwater crab species (d'Udekem d'Acoz 1999). When compared with the recent brachyuran catalogue from the Iberian Peninsula (MarcoHerrero et al. 2015), it is also noteworthy that both the Iberian and the Canarian lists comprise 20 currently accepted brachyuran superfamilies ( $\mathrm{Ng}$ et al. 2008, Spiridonov et al. 2014). The Iberian superfamilies Cyclodorippoidea, Homolodromoidea and Corystoidea do not currently occur around the Canary archipelago. The superfamily Ocypodoidea has been reported from the Canaries based on two single findings of one species. The Macaronesian superfamily Pseudozioidea, as well as the African subtropical-tropical superfamilies Trapezioidea and Cryptochiroidea are represented in Canary Island waters, but not around the Iberian Peninsula to date (Marco-Herrero et al. 2015). No freshwater crab species occur in the Canaries.

\section{REMARKS}

\section{Systematic and nominal changes since 1995}

Systematic changes have affected the taxonomical arrangement of Brachyura by Zariquiey Álvarez (1968) and Manning and Holthuis (1981), which were followed in the Canarian catalogue by González (1995). The main changes in the systematics of brachyuran crabs after those impressive catalogues were recently explained by Marco-Herrero et al. (2015).

So, instead of the $20 \mathrm{crab}$ families and 67 genera considered in González (1995), a total of 39 brachyuran families and 77 genera are presented here. Several currently accepted families were included within other families in González (1995), as follows: Ethusidae within the Dorippidae; Eriphiidae, Oziidae, Pilumnidae, Pseudoziidae, Domeciidae, and Panopeidae within the Xanthidae; Euryplacidae within the Goneplacidae; Progeryonidae within the Geryonidae; Epialtidae and Inachidae within the Majidae; Carcinidae and Polybiidae within the Portunidae; Percnidae, Plagusiidae, and Varunidae as subfamilies within the Grapsidae. The family Cryptochiridae was reported as Hapalocarcinidae in González (1995). Ocypodidae (Castro 2012) and Varunidae (Moro et al. 2014) were reported based on very few findings of few individuals. The western
Atlantic family Mathildellidae (Fransen and González in prep.) and the Thiidae (Monterroso and González in prep.) are newly reported from the Canaries here.

The current account of brachyuran crabs of the Canary Islands adds another 22 to the 108 valid species in González (1995). According to d'Udekem d'Acoz (1999), Homologenus rostratus (A. Milne-Edwards, 1880) (Bouvier 1922) is a misidentification with $\mathrm{Ho}$ mologenus boucheti Guinot and Richer de Forges, 1995. Maja crispata Risso, 1827 (Herrera et al. 1993, as M. crisperata) should not be considered here because it is a misidentification with Pisa tetraodon. Macropodia aegyptia $(\mathrm{H}$. Milne-Edwards, 1834) should be considered a synonym of Macropodia deflexa Forest, 1978. Portunus sp. in González (1995) should be assigned to Laleonectes vocans (A. Milne-Edwards, 1878). According to Reuschel and Schubart (2006), Xantho incisus Leach, 1814 is considered here a synonym of $X$. hydrophilus (Herbst, 1790), and the erroneous citation of the Indo-Pacific crab Lophozozymus incisus (H. Milne-Edwards, 1834) by Moro et al. (2014) is based on a secondary homonym of Xantho incisus, the true record. According to Neumann (1996), records for Maja squinado (Herbst, 1788) from the Canaries, the type locality, should be reassigned to Maja brachydactyla Balss, 1922. Pinnotheres pinnotheres (Linnaeus, 1758) is now considered as a new combination, Nepinnotheres pinnotheres (Linnaeus, 1758), made by Manning (1993) when he described a new genus (Nepinnotheres) with several previously Pinnotheres species.

After González (1995), Chaceon inglei Manning and Holthuis, 1989 was reported from the Canaries by Araújo et al. (2009) as a result of deep sea surveys with selective crab traps. Ebalia tuberculata Miers, 1881 was reported from both littoral (Quiles et al. 2002) and bathyal bottoms (Moro et al. 2014). Ebalia tumefacta (Montagu, 1808) was first recorded from the Canaries by Quiles et al. (2002). Some littoral crab species have recently been found by expert scuba divers and reported by Moro et al. (2014): Xaiva mcleayi (Barnard, 1947), Liocarcinus pusillus (Leach, 1816), and Pinnotheres pisum (Linnaeus, 1767). Other littoral species were reported based on dredging sampling: Macropodia linaresi Forest and Zariquiey Álvarez, 1964, Liocarcinus navigator (Herbst, 1794), and Thia scutellata (Fabricius, 1793) (Monterroso and González in prep.).

Some additional species are now present in Canarian waters, probably due to natural range expansions from nearby eastern Atlantic or Mediterranean areas: Calappa galloides Stimpson, 1859 (González et al. 2000), Ebalia tuberculata Miers, 1881 (Quiles et al. 2002), Merocryptus boletifer A. Milne-Edwards and Bouvier, 1894, and Macropipus tuberculatus (Roux, 1830) (Moro et al. 2014). However, the finding of one specimen of Neopilumnoplax sp. in Canarian waters (Fransen and González in prep.) may represent a recent colonization from the subtropical western Atlantic, or reflect that the deep-water crabs Neopilumnoplax americana (Rathbun, 1898) or Neopilumnoplax gervaini Tavares and Guinot, 1996 are amphi-Atlantic species. Regarding the specimens of Brachynotus 
Table 1. - List of the brachyuran species occurring in waters of the Canary Islands. Spatial distribution, benthic habitat, bathymetric range (depth interval in $\mathrm{m}$ known at the Canaries and elsewhere), and biogeographic pattern are provided for each species. Their occurrence (X) or absence (-) around Madeira and/or the Cape Verde Islands is also provided. Species: * doubtful presence, ** probable occurrence. Depth boundaries: ST shelf and transition area between shelf and slope (0-300 m), US upper slope (301-800 m), MS middle slope (801-2000 m), LS lower slope $(>2000 \mathrm{~m})$. Species' biogeographic pattern codes: COSM cosmopolitan or worldwide, PANT pantropical or circumtropical, AAWD amphi-Atlantic of wide distribution, AAWA amphi-Atlantic of warm affinity, EAWD eastern Atlantic of wide distribution, EACT eastern Atlantic cold-temperate, EAWT eastern Atlantic warm-temperate, ATLM Atlanto-Mediterranean, TSEA tropical and subtropical eastern Atlantic, ECAI eastern-central Atlantic island (from Azores to Cape Verde Is., and even to St. Helena, MAC Macaronesian (Azores, Madeira, Savage, and/or the Canaries).

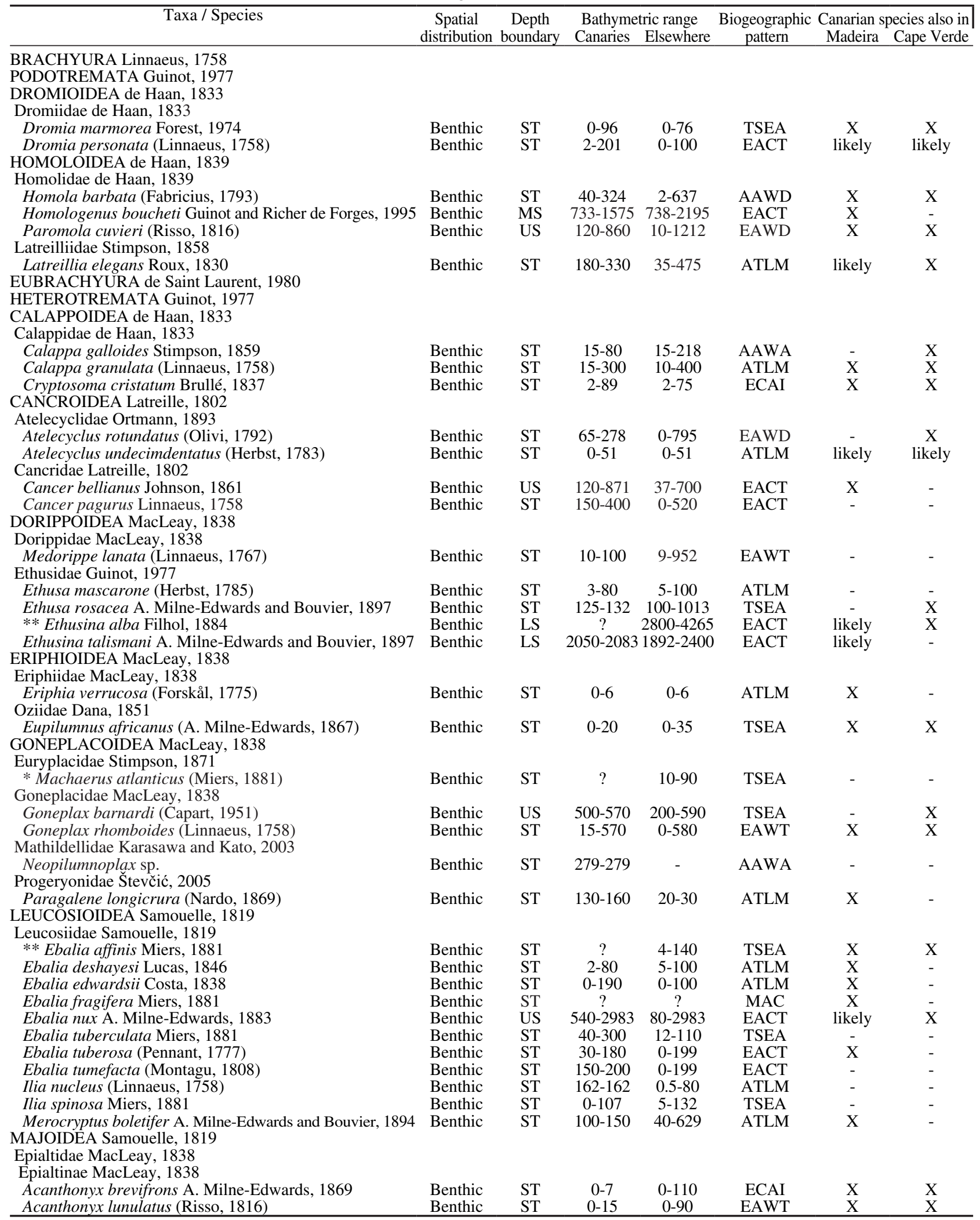




\begin{tabular}{|c|c|c|c|c|c|c|c|}
\hline \multirow{2}{*}{ Taxa / Species } & \multirow{2}{*}{$\begin{array}{c}\text { Spatial } \\
\text { distribution }\end{array}$} & \multirow{2}{*}{$\begin{array}{l}\text { Depth } \\
\text { boundary }\end{array}$} & \multicolumn{2}{|c|}{ Bathymetric range } & \multirow{2}{*}{$\begin{array}{l}\text { Biogeographic } \\
\text { pattern }\end{array}$} & \multicolumn{2}{|c|}{ Canarian species also in } \\
\hline & & & Canaries & Elsewhere & & Madeira & Cape Verde \\
\hline Pisinae Dana, 1851 & & & & & & & \\
\hline Anamathia rissoana (Roux, 1828) & Benthic & US & $100-500$ & $100-730$ & ATLM & $\mathrm{X}$ & - \\
\hline Herbstia condyliata (Fabricius, 1787) & Benthic & ST & $0-54$ & $0-80$ & ATLM & $\mathrm{X}$ & - \\
\hline Herbstia rubra A. Milne-Edwards, 1869 & Benthic & ST & $0-20$ & $0-75$ & TSEA & - & $\mathrm{X}$ \\
\hline Micropisa ovata Stimpson, 1858 & Benthic & ST & $0-3$ & $0-110$ & TSEA & - & $\mathrm{X}$ \\
\hline Pisa armata (Latreille, 1803) & Benthic & ST & $41-82$ & $18-162$ & EAWT & likely & $\mathrm{X}$ \\
\hline Pisa carinimana Miers, 1879 & Benthic & ST & $4-120$ & $4-100$ & EAWT & $X$ & - \\
\hline Pisa nodipes (Leach, 1815) & Benthic & ST & $3-70$ & $0-100$ & ATLM & $\mathrm{X}$ & $\mathrm{X}$ \\
\hline Pisa tetraodon (Pennant, 1777) & Benthic & ST & $2-50$ & $0-50$ & ATLM & - & - \\
\hline Rochinia carpenteri (Thomson, 1873) & Benthic & MS & $500-1059$ & $400-1340$ & EACT & $X$ & - \\
\hline Inachidae MacLeay, 1838 & & & & & & & \\
\hline Achaeus cranchii Leach, 1817 & Benthic & ST & $0->20$ & $0-70$ & ATLM & X & - \\
\hline Dorhynchus thomsoni Thomson, 1873 & Benthic & MS & $570-1163$ & $106-2080$ & COSM & likely & $\mathrm{X}$ \\
\hline Inachus aguiarii de Brito Capello, 1876 & Benthic & ST & $55-110$ & $20-100$ & ATLM & $\mathrm{X}$ & - \\
\hline Inachus dorsettensis (Pennant, 1777) & Benthic & US & $540-540$ & $0-749$ & EAWD & - & - \\
\hline Inachus grallator Manning and Holthuis, 1981 & Benthic & ST & $60-125$ & $36-325$ & TSEA & - & - \\
\hline Inachus nanus Manning and Holthuis, 1981 & Benthic & ST & $45-80$ & $29-118$ & TSEA & - & - \\
\hline Inachus phalangium (Fabricius, 1775) & Benthic & ST & $0.5-35$ & $0.5-160$ & EACT & $\mathrm{X}$ & - \\
\hline Inachus thoracicus Roux, 1830 & Benthic & ST & $30-90$ & $10-200$ & ATLM & - & - \\
\hline Macropodia deflexa Forest, 1978 & Benthic & ST & $23-23$ & 0-90 & EACT & - & - \\
\hline Macropodia linaresi Forest and Zariquiey Álvarez, 1964 & Benthic & ST & $5-50$ & $3.5-140$ & ATLM & - & - \\
\hline Macropodia aff. hesperiae Manning and Holthuis, 1981 & Benthic & MS & $821-821$ & - & MAC & - & - \\
\hline Macropodia longirostris (Fabricius, 1775) & Benthic & ST & $30-60$ & $4-130$ & ATLM & - & - \\
\hline Macropodia aff. longirostris (Fabricius, 1775) & Benthic & ST & $50-60$ & - & MAC & - & - \\
\hline Macropodia aff. parva van Noort and Adema, 1985 & Benthic & ST & $25-82$ & $20-90$ & TSEA & - & - \\
\hline Macropodia rostrata (Linnaeus, 1761) & Benthic & ST & $0-100$ & $0-193$ & EAWD & likely & - \\
\hline Macropodia spec. 2 Fransen, 1991 & Benthic & ST & $86-200$ & $140-170$ & MAC & - & - \\
\hline Stenorhynchus lanceolatus (Brullé, 1837) & Benthic & ST & $2-273$ & $5-96$ & TSEA & $X$ & $X$ \\
\hline Majidae Samouelle, 1819 & & & & & & & \\
\hline Eurynome aspera (Pennant, 1777) & Benthic & ST & $10-200$ & $10-1216$ & EAWD & $\mathrm{X}$ & $\mathrm{X}$ \\
\hline Maja brachydactyla Balss, 1922 & Benthic & ST & $0-72$ & 0-91 & EAWT & $X$ & - \\
\hline Maja goltziana d'Oliveira, 1888 & Benthic & ST & $50-287$ & $27-250$ & ATLM & - & - \\
\hline Oregoniidae Garth, 1958 & & & & & & & \\
\hline Ergasticus clouei A. Milne-Edwards, 1882 & Benthic & US & $420-570$ & $70-1000$ & ATLM & $\mathrm{X}$ & $\mathrm{X}$ \\
\hline PALICOIDEA Bouvier, 1898 & & & & & & & \\
\hline Palicidae Bouvier, 1898 & & & & & & & \\
\hline Palicus caronii (Roux, 1828) & Benthic & ST & $20-220$ & $18-220$ & ATLM & $X$ & $\mathrm{X}$ \\
\hline PARTHENOPOIDEA MacLeay, 1838 & & & & & & & \\
\hline Parthenopidae MacLeay, 1838 & & & & & & & \\
\hline Distolambrus maltzami (Miers, 1881) & Benthic & ST & $45-125$ & $22-550$ & EAWT & likely & $\mathrm{X}$ \\
\hline Parthenopoides massena (Roux, 1830) & Benthic & ST & $25-90$ & $3-141$ & ATLM & $\mathrm{X}$ & $\mathrm{X}$ \\
\hline Spinolambrus macrochelos (Herbst, 1790) & Benthic & ST & $100-475$ & $5-1478$ & ATLM & likely & $\mathrm{X}$ \\
\hline Velolambrus expansus (Miers, 1879) & Benthic & ST & $2-125$ & $30-170$ & ATLM & $\mathrm{X}$ & $\mathrm{X}$ \\
\hline PILUMNOIDEA Samouelle, 1819 & & & & & & & \\
\hline Pilumnidae Samouelle, 1819 & & & & & & & \\
\hline Pilumnus hirtellus (Linnaeus, 1761) & Benthic & ST & $0-25$ & $0-90$ & EACT & $\mathrm{X}$ & $\mathrm{X}$ \\
\hline Pilumnus inermis A. Milne-Edwards and Bouvier, 1894 & Benthic & ST & $200-250$ & $5-400$ & ATLM & $\mathrm{X}$ & $\mathrm{X}$ \\
\hline Pilumnus spinifer H. Milne-Edwards, 1834 & Benthic & ST & $0-20$ & $1-179$ & ATLM & $\mathrm{X}$ & $\mathrm{X}$ \\
\hline Pilumnus villosissimus (Rafinesque, 1814) & Benthic & ST & $0-20$ & $0-20$ & ATLM & $\mathrm{X}$ & - \\
\hline PORTUNOIDEA Rafinesque, 1815 & & & & & & & \\
\hline Carcinidae MacLeay, 1838 & & & & & & & \\
\hline * Carcinus aestuarii Nardo, 1847 & Benthic & ST & $10-10$ & $1-26$ & COSM & - & - \\
\hline * Carcinus maenas (Linnaeus, 1758) & Benthic & ST & $?$ & $0-60$ & EACT & - & - \\
\hline Portumnus latipes (Pennant, 1777) & Benthic & ST & $0-3$ & $0-30$ & ATLM & likely & - \\
\hline Xaiva mcleayi (Barnard, 1947) & Benthic & ST & $4-4$ & $8-73$ & TSEA & - & - \\
\hline Geryonidae Colosi, 1923 & & & & & & & \\
\hline Chaceon affinis (A. Milne-Edwards and Bouvier, 1894) & Benthic & US & $411-1350$ & $130-2047$ & EACT & $\mathrm{X}$ & $\mathrm{X}$ \\
\hline Chaceon inglei Manning and Holthuis, 1989 & Benthic & LS & $2156-2156$ & $1640-2500$ & EACT & $\mathrm{X}$ & - \\
\hline Chaceon maritae (Manning and Holthuis, 1981) & Benthic & US & ? & $100-1000$ & TSEA & - & $\mathrm{X}$ \\
\hline Geryon trispinosus (Herbst, 1803) & Benthic & US & $639-833$ & $32-2220$ & EACT & - & - \\
\hline Pirimelidae Alcock, 1899 & & & & & & & \\
\hline Pirimela denticulata (Montagu, 1808) & Benthic & ST & $0-15$ & $0-250$ & EACT & $X$ & $\mathrm{X}$ \\
\hline Polybiidae Ortmann, 1893 & & & & & & & \\
\hline Bathynectes longipes (Risso, 1816) & Benthic & ST & $30-100$ & $15-226$ & ATLM & $X$ & - \\
\hline Bathynectes maravigna (Prestandrea, 1839) & Benthic & US & $366-846$ & $60-1410$ & EACT & - & - \\
\hline Liocarcinus corrugatus (Pennant, 1777) & Benthic & ST & $1-225$ & $1-147$ & EAWT & $\mathrm{X}$ & $\mathrm{X}$ \\
\hline Liocarcinus depurator (Linnaeus, 1758) & Benthic & ST & $45-90$ & $1-871$ & EACT & - & - \\
\hline Liocarcinus holsatus (Fabricius, 1798) & Benthic & ST & $<100$ & $1-400$ & EACT & likely & - \\
\hline Liocarcinus navigator (Herbst, 1794) & Benthic & ST & $5-50$ & $0-108$ & EACT & - & - \\
\hline Liocarcinus pusillus (Leach, 1816) & Benthic & ST & $20-30$ & $0-455$ & EACT & likely & - \\
\hline Liocarcinus vernalis (Risso, 1816) & Benthic & ST & $35-100$ & $0-150$ & ATLM & - & - \\
\hline Liocarcinus zariquieyi Gordon, 1968 & Benthic & ST & $23-80$ & $5-80$ & ATLM & - & - \\
\hline Macropipus tuberculatus (Roux, 1830) & Benthic & ST & $100-150$ & $48-748$ & EACT & likely & - \\
\hline Polybius henslowii Leach, 1820 & Pelagic & - & $0-5$ & $2-1245$ & EACT & likely & - \\
\hline Portunidae Rafinesque, 1815 & & & & & & & \\
\hline Portuninae Rafinesque, 1815 & & & & & & & \\
\hline Laleonectes vocans (A. Milne-Edwards, 1878) & Benthic & ST & $5-10$ & $6-37$ & AAWA & $\mathrm{X}$ & - \\
\hline
\end{tabular}




\begin{tabular}{|c|c|c|c|c|c|c|c|}
\hline \multirow{2}{*}{ Taxa / Species } & \multirow{2}{*}{$\begin{array}{c}\text { Spatial } \\
\text { distribution }\end{array}$} & \multirow{2}{*}{$\begin{array}{c}\text { Depth } \\
\text { boundary }\end{array}$} & \multicolumn{2}{|c|}{ Bathymetric range } & \multirow{2}{*}{$\begin{array}{l}\text { Biogeographic } \\
\text { pattern }\end{array}$} & \multicolumn{2}{|c|}{ Canarian species also in } \\
\hline & & & & Elsewhere & & Madeira & Cape Verde \\
\hline Portunus (Portunus) hastatus (Linnaeus, 1767) & Benthic & ST & $2-60$ & $2-40$ & TSEA & $\mathrm{X}$ & $\mathrm{X}$ \\
\hline Portunus (Portunus) inaequalis (Miers, 1881) & Benthic & ST & $?$ & $4-73$ & TSEA & $\mathrm{X}$ & $\mathrm{X}$ \\
\hline Thalamitinae Paul'son, 1875 & & & & & & & \\
\hline Thalamita poissonii (Audouin, 1826) & Benthic & ST & $0.5-120$ & $0.5-20$ & PANT & - & $\mathrm{X}$ \\
\hline Thiidae Dana, 1852 & & & & & & & \\
\hline Pseudoziidae MacLeay, 1838 & & & & & & & \\
\hline $\begin{array}{l}\text { Euryozius bouvieri (A. Milne-Edwards, 1869) } \\
\text { TRAPEZIOIDEA Miers, } 1886\end{array}$ & Benthic & ST & $0-23$ & $6-30$ & ECAI & $\mathrm{X}$ & $\mathrm{X}$ \\
\hline Domeciidae Ortmann, 1893 & & & & & & & \\
\hline $\begin{array}{l}\text { Domecia acanthophora africana Guinot, } 1964 \\
\text { XANTHOIDEA MacLeav } 1838\end{array}$ & Benthic & ST & $0-0$ & $0-35$ & ECAI & - & $\mathrm{X}$ \\
\hline $\begin{array}{l}\text { Panopeidae Ortmann, } 1893 \\
\text { Panopeus africanus A. Milne-Edwards } 1867\end{array}$ & Benthic & ST & $0-8$ & $0-140$ & TSEA & - & $\mathrm{X}$ \\
\hline Xanthidae MacLeay, 1838 & & & & & & & \\
\hline $\begin{array}{l}\text { Actaeinae Alcock, } 1898 \\
\text { Paractaea monodi Guinot, } 1969\end{array}$ & Benthic & ST & $5-82$ & $0-200$ & ATI M & $\mathrm{X}$ & \\
\hline Paractaea rufopunctata (H. Milne-Edwards, 1834) & Benthic & ST & $0-45$ & $0-91$ & PANT & - & $\begin{array}{c}X \\
-\end{array}$ \\
\hline Euxanthinae Alcock, 1898 & & & & & & & \\
\hline Glyptoxanthus cavernosus (A. Milne-Edwards, 1878) & Benthic & ST & $0-0$ & $0-17$ & ECAI & - & $\mathrm{X}$ \\
\hline Monodaeus couchii (Couch, 1851) & Benthic & ST & $20-500$ & $0-1415$ & EAWT & likely & $\mathrm{X}$ \\
\hline ** Monodaeus rouxi (Capart, 1951) & Benthic & ST & $?$ & $11-510$ & TSEA & $\mathrm{X}$ & - \\
\hline Xanthinae MacLeay, 1838 & & & & & & & \\
\hline Coralliope parvula (A. Milne-Edwards, 1869) & Benthic & ST & $0-33$ & $0-355$ & TSEA & - & $\mathrm{X}$ \\
\hline Microcassiope minor (Dana, 1852) & Benthic & ST & $0-20$ & $0-220$ & AAWA & $\mathrm{X}$ & $\mathrm{X}$ \\
\hline Nanocassiope melanodactyla (A. Milne-Edwards, 1867) & Benthic & ST & $5-110$ & $5-225$ & TSEA & $\mathrm{X}$ & $\mathrm{X}$ \\
\hline Xantho hydrophilus (Herbst, 1790) & Benthic & ST & $0-25$ & $0-37$ & ATLM & $\mathrm{X}$ & $\mathrm{X}$ \\
\hline Xantho pilipes A. Milne-Edwards, 1867 & Benthic & ST & $0-36$ & $0-133$ & EAWD & $\mathrm{X}$ & - \\
\hline Xantho poressa (Olivi, 1792) & Benthic & ST & $0-15$ & $0-20$ & ATLM & - & - \\
\hline Xantho sexdentatus (Miers, 1881) & Benthic & ST & $0-15$ & $0-35$ & TSEA & likely & $\mathrm{X}$ \\
\hline Xantho sp. Fransen, 1991 & Benthic & ST & $0-7$ & $0-15$ & TSEA & $\mathrm{X}$ & $\mathrm{X}$ \\
\hline Zosiminae Alcock, 1898 & & & & & & & \\
\hline Platypodiella picta (A. Milne-Edwards, 1869) & Benthic & ST & $0-30$ & $0-30$ & TSEA & $\mathrm{X}$ & - \\
\hline THORACOTREMATA Guinot, 1977 & & & & & & & \\
\hline CRYPTOCHIROIDEA Paul'son, 1875 & & & & & & & \\
\hline Cryptochiridae Paul'son, 1875 & & & & & & & \\
\hline Detocarcinus balssi (Monod, 1956) & Benthic & ST & $20-25$ & $3-62$ & TSEA & - & - \\
\hline GRAPSOIDEA MacLeay, 1838 & & & & & & & \\
\hline Grapsidae MacLeay, 1838 & & & & & & & \\
\hline Grapsus adscensionis (Osbeck, 1765) & Benthic & ST & $0-7$ & $0-4$ & TSEA & $\mathrm{X}$ & $\mathrm{X}$ \\
\hline Pachygrapsus marmoratus (Fabricius, 1787) & Benthic & ST & $0-6$ & $0-20$ & ATLM & $\mathrm{X}$ & - \\
\hline Pachygrapsus maurus (Lucas, 1846) & Benthic & ST & $0-6$ & $0-6$ & ATLM & $\mathrm{X}$ & $\mathrm{X}$ \\
\hline Pachygrapsus transversus (Gibbes, 1850) & Benthic & ST & $0-7$ & $0-7$ & PANT & $\mathrm{X}$ & $\mathrm{X}$ \\
\hline Planes minutus (Linnaeus, 1758) & Pelagic & - & $0-31$ & $0-31$ & PANT & $\mathrm{X}$ & $\mathrm{X}$ \\
\hline Percnidae Števčić, 2005 & & & & & & & \\
\hline Percnon gibbesi (H. Milne Edwards, 1853) & Benthic & ST & $0-29$ & $5-29$ & PANT & $\mathrm{X}$ & $\mathrm{X}$ \\
\hline Plagusiidae Dana, 1851 & & & & & & & \\
\hline Euchirograpsus liguricus H. Milne-Edwards, 1853 & Benthic & ST & $150-250$ & $10-359$ & EAWT & $\mathrm{X}$ & $\mathrm{X}$ \\
\hline Plagusia depressa (Fabricius, 1775) & Benthic & ST & $0-5$ & $0-5$ & AAWA & $\mathrm{X}$ & $\mathrm{X}$ \\
\hline Varunidae H. Milne-Edwards, 1853 & & & & & & & \\
\hline Brachynotus sexdentatus (Risso, 1827) & Benthic & ST & $1.5-1.5$ & $0-2$ & ATLM & - & - \\
\hline OCYPODOIDEA Rafinesque, 1815 & & & & & & & \\
\hline Ocypodidae Rafinesque, 1815 & & & & & & & \\
\hline Uca (Afruca) tangeri (Eydoux, 1835) & Benthic & ST & $0-2$ & $0-2$ & TSEA & - & $\mathrm{X}$ \\
\hline PINNOTHEROIDEA de Haan, 1833 & & & & & & & \\
\hline Pinnotheridae de Haan, 1833 & & & & & & & \\
\hline Nepinnotheres pinnotheres (Linnaeus, 1758) & Benthic & ST & $2-25$ & $6-250$ & ATLM & - & - \\
\hline Pinnotheres pisum (Linnaeus, 1767) & Benthic & ST & $38-38$ & $0-150$ & EACT & - & - \\
\hline
\end{tabular}

sexdentatus (Risso, 1827) found in a brackish littoral pond with mobile sandy substrate (Charca de Maspalomas, S of Gran Canaria), under stones at $1.5 \mathrm{~m}$ of depth (Moro et al. 2014), given that this species has been introduced in other zones (for instance, the U.K.) and that even the habitat observed is not the more appropriate for this species, the most plausible reason for that finding is a human-mediated activity (through ballast water, fouling or oil platform, or a release from aquarium or pet trade).

The finding in 2011 of one single male of $U c a$ (Afruca) tangeri (Eydoux, 1835) on a sandstone platform on a crowded urban beach (Las Canteras, Gran
Canaria) could be a consequence of larvae drifting transport between the African coast and the Canary archipelago, and the subsequent process of settlement and growing of an isolated individual (Castro 2012). Another plausible explanation is a human-mediated introduction (release from aquarium or pet trade).

Acanthonyx brevifrons A. Milne-Edwards, 1869 is first recorded from the Canary Islands herein, based on one male (ICCM408, $3.8 \mathrm{~mm}$ carapace length) collected by hand at the Bay of Melenara, Gran Canaria, $27^{\circ} 59^{\prime} \mathrm{N} 15^{\circ} 12^{\prime} \mathrm{W}$, Dec. 1997, on red algae Gelidium, 0-7 m. An eastern Atlantic species, appearing to be endemic from the NE Atlantic archipelagos of Azores 
(Milne-Edwards and Bouvier 1894), Madeira (Chapman and Santler 1955, as A. lunulatus var. brevifrons) and the Cape Verde Islands (Milne-Edwards 1869, type locality), associated with Cystoseira meadows or rocks with algae, from the intertidal to up more than 110 m of depth (d'Udekem d'Acoz 1999).

Although Forest and Zariquiey Álvarez (1964) reviewed the Mediterranean species of Macropodia Leach, 1814 and their nomenclature, specific identification of the eastern-central Atlantic Macropodia has remained particularly difficult (Manning and Holthuis 1981, d'Udekem d'Acoz 1999). Eight Macropodia species not fully identified at specific level from the Canary-Cape Verde region were included in the Fransen (1991) account. Of them, four species were compiled in the d'Udekem d'Acoz (1999) inventory, with three of them (M. aff. hesperiae, $M$. aff. longirostris, and Macropodia spec. 2) potentially representing endemic forms from the Macaronesia.

\section{Species of doubtful presence in the Canary Islands}

Machaerus atlanticus (Miers, 1881) has only been recorded from the Canary Islands by Barquín-Diez and Carrillo (1988) (as Pilumnoplax atlantica, with no collecting data). In the Eastern Atlantic, it is known from Senegal to Congo-Brazzaville, on mixed bottoms dominated by mud, 10-90 m (d'Udekem d'Acoz 1999).

From the time when Santaella (1973) conducted his research, confusion has long existed over the validity of records for the species of Carcinus from the Canaries. Unfortunately, neither González (1995) nor this work have shed too much light on this issue. Carcinus maenas (Linnaeus, 1758) was first recorded from the Canaries by Heller (1863) (with no location), and then compiled by García Cabrera (1971) ("Canaries"). Santaella (1973) did not give validity to these citations based on the absence of a well-documented finding, and also the fact that the European species of Carcinus had not yet been separated in the 19th century. Although live specimens of this species were recorded as commercialized bait (Núñez et al. 2011), they were imported. The species' supposed natural range of distribution covered the eastern Atlantic, from SE Iceland to Atlantic Morocco, the Western Sahara and Mauritania, and it is also known from the western Mediterranean. However, it has been introduced in the NE Pacific, South Africa, S of Australia, and perhaps in the NW Atlantic and has been accidentally observed in many locations around the world (d'Udekem d'Acoz 1999). It is found in intertidal and shallow subtidal zones, 0-60 m, mainly at 0-6 m, rarely to $200 \mathrm{~m}$ (?), under rocks and algae, tide pools, marshes and seagrass beds, and in low to full salinity areas (d'Udekem d'Acoz 1999, Fransen 2015a in WoRMS). Carcinus aestuarii Nardo, 1847 was probably first recorded from the Canaries by Heller (1863) (as C. maenas) and Almaça (1960), and then compiled by Zariquiey Álvarez (1968) (as C. mediterraneus), García Cabrera (1971) (as C. maenas), and Manning and Holthuis (1981) among others. The presence of this species in waters of the Canaries was corroborated by Barquín-Diez et al. (1982-1983) (one spec., Tenerife, muddy sand, $10 \mathrm{~m}$ ), and collected again on polluted bottoms of the Port of Santa Cruz de Tenerife (González 1995, based on a pers. comm.). Were both specimens well identified? If correctly identified, were both specimens introduced? It has been recorded in scattered localities worldwide: the West Pacific (Japan, introduced, d'Udekem d'Acoz 1999), the Indo-West Pacific, the Indian Ocean (Suez Canal, ?Red Sea), the western Atlantic (USA). It has been found in the eastern Atlantic, the Mediterranean and Black Seas, in Morocco, Mauritania, and South Africa (Fransen 2015b in WoRMS), on muddy sand, under rocks, and seagrass meadows, at 1-26 m (Abelló et al. 1988, d'Udekem d'Acoz 1999). If C. aestuarii is the right identification for the species occurring in the Canaries, it should be considered as introduced. If $C$. maenas occurs in the Canaries, this would represents a range extension of this west-African species.

\section{Species probably occurring in the Canary Islands}

Ethusina alba Filhol, 1884: an eastern Atlantic species, found between France and the Azores, off Azores and off Cape Verde Islands, on mud bottoms often with pumice stone, 2800-4265 m (Manning and Holthuis 1981, Fransen 1991). Ebalia affinis Miers, 1881: an eastern Atlantic species, known from Seine Bank, N of Madeira (Doflein 1904), the Cape Verde Islands (Monod 1956) and from Senegal to Angola, including São Tomé and Principe Islands (Manning and Holthuis, 1981), on calcareous algae and mud with shells, 4-140 m (d'Udekem d'Acoz 1999). Portunus (Portunus) inaequalis (Miers, 1881): an eastern Atlantic species, known from Madeira (Türkay 1976), the Cape Verde Islands and Guinea-Bissau southward to Angola, including the islands of the Gulf of Guinea, on various bottom types, 4-73 m, mainly at 4-40 m (Manning and Holthuis 1981, d'Udekem d'Acoz 1999). To date no records for these three crab species exist from the Canaries. However, as they have been recorded from northern and southern adjacent areas, their presence in the Canary Islands is very probable. Very recently, photographic evidence was provided to me proving the occurrence of large specimens of $P$. $(P$.) inaequalis in waters of both Tenerife and Gran Canaria islands.

Monodaeus rouxi (Capart, 1951): an eastern Atlantic species, found in Madeira (Fransen 1991), Senegal, Gambia and southward to Angola (d'Udekem d'Acoz 1999), mainly on mud, also on sand and clay (Forest and Guinot 1966, Fransen 1991), from $11 \mathrm{~m}$ (Manning and Holthuis 1981) to $510 \mathrm{~m}$ depth (Fransen 1991). No record exists for this species from the Canaries. However, it has been recorded from Madeira and the neighbouring African coast, so its presence in the Canary Islands is very probable.

Cancer pagurus Linnaeus, 1758: an eastern Atlantic species, known from Norway $\left(70^{\circ} \mathrm{N}\right)$ to S Portugal, including the Mediterranean (very rare), rocks and sand, from the intertidal to $520 \mathrm{~m}$ of depth, rarely down to $100 \mathrm{~m}$ (d'Udekem d'Acoz 1999). Some doubtful findings from Canada, USA and the Pacific Ocean 
have been reported in global databases. Macaronesia: Azores (Borges et al. 2010). Up to three fishermen have reported to us on sporadic catches of ox crab (= 'buey liso' in Canarian Spanish), few individuals per trap, off Fuerteventura. Some of these catches were sold in local supermarkets, and a dried carapace used as an ornamental object was examined by us. So the northeastern sector of the Canaries seems to be the southern distribution limit for the species, coinciding with the coldest waters around the archipelago.

\section{Spatial and vertical distribution}

Of the 132 brachyuran species occurring around the Canary Islands, 130 are benthic forms (Table 1). Polybius henslowii has been reported as epi- to bathypelagic, with benthic stages (e.g. González-Gurriarán 1987). Planes minutus is epipelagic, living on loggerhead sea turtles and inanimate flotsam (e.g. Dellinger et al. 1997), but also has benthic stages when flotsam reaches the beaches. Dorhynchus thomsoni is known to be benthic with a pelagic initial stage (e.g. Hartnoll et al. 1987). Portunus (Portunus) hastatus has juveniles with pelagic stages, but adults are clearly benthic (Table 1).

Of the 130 brachyuran benthic species occurring in the Canaries, 112 are forms of the shelf and transition area between shelf and slope (ST, 86.2\%, mainly inhabiting at a depth interval of 0-300 m), 11 are upper slope species (US, 8.5\%, mainly at a depth interval of 301-800 m), 4 are middle-slope species (MS, 3.1\%, mainly at a depth interval of 801-2000 m), and 3 are lower slope species (LS, 2.3\%, mainly at a depth greater than $2000 \mathrm{~m}$ ) (Table 1).

Seven brachyuran benthic species seem to occur in waters of the Canary Islands at the shallowest depth ever recorded elsewhere: Homologenus boucheti, Ilia spinosa, Velolambrus expansus, Xaiva mcleayi, Laleonectes vocans, Euryozius bouvieri, and Percnon gibbesi. Seventeen brachyuran benthic species seem to occur in waters of the Canary Islands at the greatest depth ever recorded elsewhere: Dromia marmorea, $D$. personata, Cryptosoma cristatum, Cancer bellianus, Paragalene longicrura, Ebalia edwardsii, E. tuberculata, E. tumefacta, Ilia nucleus, Pisa carinimana, Inachus aguiarii, Stenorhynchus lanceolatus, Maja goltziana, Liocarcinus corrugatus, L. zariquieyi, Portunus (Portunus) hastatus and Thalamita poissonii.

\section{Zoogeographic considerations}

To describe for the first time the different components of the brachyuran fauna, all the Canarian brachyuran crabs listed herein (132 species) are grouped by biogeographic pattern in Table 2 . A first preliminary description showed three main groups: 39 ATLM species (29.5\%), 28 TSEA species (21.2\%), and 27 EACT species $(20.5 \%)$. The remaining eight biogeographic groups varied from six EAWD species $(4.5 \%)$ to one AAWD species $(0.8 \%)$ (Table 2$)$.

The presence of one MAC endemic species, $M a$ cropodia aff. hesperiae Manning and Holthuis, 1981, is noteworthy. The only record for this middle-slope species from the Canary Islands was by González (1995), based on one specimen caught off Playa de Santiago, S of La Gomera, $821 \mathrm{~m}$, which was identified by C.H.J.M. Fransen, and then validated by d'Udekem d'Acoz (1999). Since all Macropodia species known to date were discarded during the identification, the Canarian specimen may represent a new species, even being an endemism from the Canary Islands or the Macaronesia. The true Macropodia hesperiae Manning and Holthuis, 1981 inhabits littoral waters at depths between 46 and $97 \mathrm{~m}$ from Senegal to Nigeria (Manning and Holthuis 1981).

The Canarian brachyuran crabs inhabiting the shelf and transition area between shelf and slope (123 species), i.e. those living shallower than the upper slope, at 0-300 $\mathrm{m}$, are grouped by biogeographic pattern in Table 2. This description of the benthic littoral and/or upperbathyal species showed the same three main groups: 39 ATLM species (31.7\%), 28 TSEA species (22.8\%), and 21 EACT species $(17.1 \%)$. The remaining eight biogeographic groups varied from ten EAWT species $(8.1 \%)$ to one AAWD species $(0.8 \%)$ (Table 2$)$.

The presence of four ECAI species is noteworthy. 1. Cryptosoma cristatum Brullé, 1837: known from the Azores (Wirtz and Martins 1993), Porto Santo, Madeira, Desertas Islands (Türkay 1976, as Crytosoma cristatum, erroneous spelling), the Canaries (Brullé 1837-1839, as $C$. cristata, type locality), the Cape Verde Islands (Monod 1956), and St. Helena Island; as an introduced species, found in the southwestern Mediterranean, with one record from the Alboran Sea (García-Raso 1993). 2. Acanthonyx brevifrons A. Milne-Edwards, 1869: from the Azores (Milne-Edwards and Bouvier 1894), Madeira (Chapman and Santler 1955, as A. lunulatus var.

Table 2. - Zoogeographic composition of the Canarian brachyuran fauna showing the number of species (N) grouped by biogeographic pattern.

\begin{tabular}{|c|c|c|c|c|}
\hline \multirow{2}{*}{ Species' biogeographic pattern } & \multicolumn{2}{|c|}{$\begin{array}{c}\text { All Canarian brachyuran } \\
(132 \text { species, depth } 0->2000 \mathrm{~m})\end{array}$} & \multicolumn{2}{|c|}{$\begin{array}{l}\text { Littoral and upper bathyal benthic } \\
(123 \text { species, depth } 0-300 \mathrm{~m})\end{array}$} \\
\hline & $\mathrm{N}$ & $\%$ & $\mathrm{~N}$ & $\%$ \\
\hline Atlanto-Mediterranean (ATLM) & 39 & 29.5 & 39 & 31.7 \\
\hline Tropical and subtropical Eastern Atlantic (TSEA) & 28 & 21.2 & 28 & 22.8 \\
\hline Eastern Atlantic cold-temperate (EACT) & 27 & 20.5 & 21 & 17.1 \\
\hline Eastern Atlantic warm-temperate (EAWT) & 10 & 7.6 & 10 & 8.1 \\
\hline Eastern Atlantic of wide distribution (EAWD) & 6 & 4.5 & 6 & 4.9 \\
\hline Amphi-Atlantic of warm affinity (AAWA) & 5 & 3.8 & 5 & 4.1 \\
\hline Eastern-central Atlantic island (ECAI) & 5 & 3.8 & 5 & 4.1 \\
\hline Pantropical or circumtropical (PANT) & 5 & 3.8 & 4 & 3.3 \\
\hline Macaronesian (MAC) & 4 & 3.0 & 3 & 2.4 \\
\hline Cosmopolitan or worldwide (COSM) & 2 & 1.5 & 1 & 0.8 \\
\hline Amphi-Atlantic of wide distribution (AAWD) & 1 & 0.8 & 1 & 0.8 \\
\hline
\end{tabular}


brevifrons), the Canaries (present work) and the Cape Verde Islands (Milne-Edwards 1869, type locality). 3. Euryozius bouvieri (Milne-Edwards, 1869): from the Azores (Barrois 1888, as Ozius Edwardsi), Madeira (Türkay 1976), the Canaries (Santaella 1973) and the Cape Verde Islands (Milne-Edwards and Bouvier 1900). 4. Glyptoxanthus cavernosus (Milne-Edwards, 1878): from the Canaries (Fransen 1991) and the Cape Verde Islands (Bouvier 1922).

The occurrence of three MAC endemics is also noteworthy. 1. Ebalia fragifera Miers, 1881: from Madeira and the Canaries (Miers 1881, type locality). To date it is only known from the type material and, according to Monod (1956), its status needs to be reviewed. 2. Macropodia aff. longirostris (Fabricius, 1775): from the British Isles, Belgian North Sea, France, and the Mediterranean and Black Seas (d'Udekem d'Acoz 1999), detrital bottoms, sand, rocks covered by algae, and seagrass meadows, $4-130 \mathrm{~m}$, mainly at $18-36 \mathrm{~m}$ (d'Udekem d'Acoz 1999). First recorded from the Canaries by Milne-Edwards and Bouvier (1900) (as Stenorhynchus aegyptius) based on "Talisman 1883" material (one spec., $28^{\circ} 49^{\prime} \mathrm{N} 13^{\circ} 53^{\prime} \mathrm{W}$, sand and rocks, $30 \mathrm{~m}$ ). This record was compiled by Bouvier (1940) but the collection was placed at a depth of $40 \mathrm{~m}$. Forest (1964) reassigned this specimen to the genus Macropodia. Forest (1978) demonstrated that H. Milne-Edwards had based the species' description on material of $M$. longirostris (Fabricius, 1775). 3. Macropodia spec. 2 Fransen, 1991: only known from the Savage Islands (Fransen 1991, one spec., Selvagem Pequena, 3001'N $16^{\circ} 00^{\prime} \mathrm{W}, 140-170 \mathrm{~m}$ ) and the Canary Islands (Fransen 1991, La Palma, one spec., 28³8'N 1759’ W, 200 m; one spec., $28^{\circ} 38^{\prime} \mathrm{N} 17^{\circ} 58^{\prime} \mathrm{W}$, mud with oysters, 86$110 \mathrm{~m}$ ). This material may represent a new species, even being an endemism from the Canary-Savage Islands or more extended in Macaronesia.

Twenty-seven brachyuran benthic species occurring in the Canary Islands currently have their southern limit of distribution in this archipelago: Cancer bellianus, Cancer pagurus, Ethusa mascarone, Paragalene longicrura, Ebalia edwardsii, Ebalia fragifera, Ebalia tuberosa (with certainty in the Canaries and the Western Sahara, Manning and Holthuis 1981), Ebalia tumefacta, Merocryptus boletifer, Anamathia rissoana, Inachus thoracicus (probably), Macropodia deflexa, Macropodia linaresi, Macropodia longirostris, Macropodia sp., Pilumnus villosissimus, Portumnus latipes, Chaceon inglei, Geryon trispinosus, Bathynectes longipes, Liocarcinus navigator, Liocarcinus holsatus, Liocarcinus zariquieyi, Macropipus tuberculatus, Xantho poressa, Pachygrapsus marmoratus and Brachynotus sexdentatus. This southern group of 27 species represents $20.8 \%$ of the 130 benthic crabs listed herein.

Another 23 brachyuran benthic species occurring in the Canary Islands currently have their northern limit of distribution in this archipelago: Calappa galloides, Ethusa rosacea, Machaerus atlanticus, Goneplax barnardi, Ebalia tuberculata, Ilia spinosa, Herbstia rubra, Micropisa ovata, Inachus grallator, Inachus nanus, Macropodia aff. parva, Stenorhynchus lanceolatus,
Palicus caronii, Velolambrus expansus, Chaceon maritae, Thalamita poissonii, Domecia acanthophora africana, Glyptoxanthus cavernosus, Coralliope parvula, Microcassiope minor, Nanocassiope melanodactyla, Xantho sexdentatus (one single record from the Gulf of Cadiz, Marco-Herrero et al. 2015) and Detocarcinus balssi. This northern group of 23 species represents $17.7 \%$ of the 130 benthic crabs listed herein.

Seventy-eight brachyuran benthic species occur in both the Canary and Madeira archipelagos (including 16 species very probably present at Madeira) (Table 1); this represents $60.0 \%$ of species shared with Madeira. Sixty-three brachyuran benthic species occur in both the Canary and Cape Verde archipelagos (including two species very probably present at the Cape Verde Islands) (Table 1); this represents $48.5 \%$ of species shared with the Cape Verde Islands. Like the Canaries, Madeira belongs to the Macaronesian ecoregion within the Lusitanian province of the Temperate Northern Atlantic realm, whereas the Cape Verde Islands belongs to its own ecoregion within the West African Transition province of the Tropical Atlantic realm (Spalding et al. 2007). The above-mentioned information could be relevant in the context of faunal movements derived from the eastern Atlantic warming.

\section{The Canarian carcinofauna: future changes}

The ongoing phylogenetic studies together with the use of larval morphology are expected to bring further changes in the systematics of brachyuran decapods; these will have an impact at several taxonomic levels, from species to superfamilies. The main changes will affect new synonymizations, the split of some taxa into new species, and the erection of new genera (e.g. Marco-Herrero et al. 2015). Therefore, significant changes in the Canarian carcinofauna are expected to come in the near future.

Some taxa occurring in waters of the Canary Islands need an in-depth revision. As mentioned, confusion exists over the status of the Carcinus records. The Leucosiidae Samouelle, 1819, in particular the genus Ebalia Leach, 1817, with the necessity to review the status of Ebalia fragifera Miers, 1881, only known from the type material, needs to be revised. The Epialtidae MacLeay, 1838, in particular the genus Pisa Leach, 1814, shows much confusion among species. The Inachidae MacLeay, 1838, in particular the genera Inachus Weber, 1795 and Macropodia Leach, 1814, need to be revised. Within the Inachus species, some geographic distribution remains uncertain due to the morphological similarity and confusion among males of these species and their respective close relatives. The Macropodia species have been traditionally assigned and reassigned to different species, but there remain some doubtful synonyms and several species affinis, i.e. with specimens apparently related to, or resembling, a known species. The Parthenopidae MacLeay, 1838 is a taxonomically problematic group. Fransen (1991) reported on intermediate forms between Parthenope miersii (A. Milne-Edwards and Bouvier, 1898) (a synonym 
of Spinolambrus macrochelos (Herbst, 1790)) and Parthenope notialis Manning and Holthuis, 1981 from Mauritania and the Cape Verde Islands. In the Geryonidae Colosi, 1923, morphological differences between the Atlantic Geryon trispinosus (Herbst, 1803) and the Mediterranean Geryon longipes A. Milne-Edwards, 1882 are not clear (d'Udekem d'Acoz 1999). The Polybiidae Ortmann, 1893, in particular the genus Liocarcinus Stimpson, 1871, is probably one of the most taxonomically complicate. Within the Domeciidae Ortmann, 1893, the status of Domecia acanthophora africana Guinot, 1964 and its parent Domecia acanthophora (Desbonne, in Desbonne and Schramm, 1867) needs to be clarified, as well as the geographic range of each taxon.

Finally, within the Xanthidae MacLeay, 1838, morphology differences and distribution of the species of genus Paractaea Guinot, 1969 should be better explained. In this regard, records from the Canaries were Brullé (1837-1839) (as Xantho rufo-punctatus), MilneEdwards and Bouvier (1900) (as Actaea rufopunctata) and Holthuis and Gottlieb (1958) for P. rufopunctata (H. Milne-Edwards, 1834); and Guinot (1969), Santaella (1974), Fransen (1991) and González (1995) for P. monodi Guinot, 1969.

Although description of new brachyuran species is not expected to occur at a significant rate, an increase in the number of species from the Canary Islands is expected to result from trawling and dredging samplings, as well as from human-mediated introduction of non-native species, mainly via the following main vectors: through ballast waters, fouling, oil platforms, and release of aquarium or pet trade. Triay-Portella et al. (2015) pointed out that a process driving massive introduction to the Canaries of non-indigenous tropical and subtropical fish species has been taking place in the last five years through oil platforms. Concerning the natural expansion of species from adjacent areas, northwestern African and Cape-Verdean warm crab species are likely candidates to expand the Canarian checklist. A tropicalization process of fish assemblages in temperate biogeographic transition zones, including the Macaronesian region and some parts of the Mediterranean Sea, has been postulated by many authors (Brito et al. 2005, Wirtz et al. 2008, Afonso et al. 2013, Horta Costa et al. 2014). This process is associated with global warming in many cases (Brito et al. 2005, Perry et al. 2005, Occhipinti-Ambrogi 2007). In temperate marine ecosystems, climatedriven change in biotic interactions can profoundly alter ecological communities, particularly when they impact foundation species (Vergés et al. 2014). In particular, the tropicalization process associated with global warming may mainly affect the presence of the 33 brachyuran benthic species occurring in the Canaries which currently have their southern limit of distribution in this archipelago. These anthropogenic vectors of introduction of warm-affinity exotic species are synergic with the natural range extensions (Triay-Portella et al. 2015), and therefore all causes together are significantly reinforcing the tropical and subtropical component of their littoral and upper- bathyal brachyuran assemblages. The ecological impact of these non-indigenous crabs on the native community remains unknown.

\section{Species of commercial interest and conservation measures}

The Brachyura may be claimed to contain the highest degree of diversity among decapod crustaceans and include both crab species with an important role in trophic webs and others of commercial interest (Marco-Herrero et al. 2015).

Several deep-water crab species, such as Cancer bellianus, C. pagurus, Maja goltziana, Chaceon affinis and Paromola cuvieri, are targeted by local smallscale fisheries operating with non-selective bottom traps at some island localities, but their catches are moderate (e.g Santaella 1973, González 1995, TriayPortella et al. 2014, Biscoito et al. 2015). Maja brachydactyla is seasonally fished with traps and sometimes with gillnets at a few localities (e.g Santaella 1973, González 1995). A dozen intertidal crab species, such as Plagusia depressa, Eriphia verrucosa, Xantho spp., Grapsus adscensionis, Pachygrapsus spp. and Percnon gibbesi, are being intensively harvested, mainly by hand by both recreational and professional fishermen, to be used for human consumption and/or as bait in the small-scale fisheries. P. gibbesi and Platypodiella picta are also collected by hand and used for both aquarium and pet trade.

The Canarian population of P. picta has been considered as potentially menaced by habitat losses and uncontrolled harvesting (aquarium, dry specimens), and its collecting is currently forbidden by the regional government. Similar situations have been observed by the author regarding $P$. depressa, E. verrucosa, Xantho spp., G. adscensionis, Pachygrapsus spp. and $P$. gibbesi, with different degrees of harvesting pressure dependent mainly on human demography and resource accessibility at each island and insular sector. On some islands the population status of some of these threatened shallow-water species is critical, even close to local extinction. Basic biological parameters of these crabs should be determined, their island populations evaluated, and some conservation measures urgently implemented in order for them to be recovered in the near future.

A panel of experts in marine organism reproduction proposed the implementation of a minimum landing size (MLS, in cm carapace length) for the following harvested or fished crab species: $P$. depressa and $G$. adscensionis $(6 \mathrm{~cm}), C$. affinis $(13 \mathrm{~cm})$ and $C$. bellianus (19 cm) (González et al. 2012b). Considering the newness of some of these local fisheries, the absence of reliable landing statistics, and a lack of knowledge pertaining to population biomasses, initial precautionary MLS have been recently proposed for $P$. cuvieri $(9.2 \mathrm{~cm})$ (Triay-Portella et al. 2014) and $C$. affinis (12.5-13.0 cm) (Biscoito et al. 2015), together with specific additional management measures. However, no conservation measures have been implemented to date by national or regional regulatory 
bodies for the sustainable use of these 18 exploited species, except an MLS for $P$. depressa $(5 \mathrm{~cm})$ and $G$. adscensionis $(6 \mathrm{~cm})$.

\section{AKNOWLEDGEMENTS}

Thanks are due to the reputed carcinologists Drs C.H.J.M. Fransen, C. d'Udekem d'Acoz, J.E. GarcíaRaso, J.I. González-Gordillo, and P. Wirtz for helping us in the identification of some specimens and/or sending scientific articles and literature of restricted use. Special thanks to the referees (Prof. Dr García-Raso and another anonymous one) for improving the manuscript. We express our gratitude to I.J. Lozano, J.M. Hernández Otero, J.I. Santana, M. Biscoito (MMF), E. Santaella, J.A. Quiles, R. Herrera, F. Espino, R. Triay-Portella (he also helped me with the map), J.G. Pajuelo, J.M. Lorenzo, J.M. Landeira, F. Tuya, N.E. González, M. Freitas, R. Araújo (MMF), F. Hernández (TFMC), A. de Vera (TFMC), A. Telle, A. Boyra, C. Fernández, J.J. Torres and R. Herrero for their assistance on board/in the laboratory and/or for providing specimens, bibliography and/or information. Some specimens and their related data were taken from our research projects DGXIV/C/1 94/034 (European Commission), CAMARON and AQUACRU (Canary Is. Government), PESCPROF 1-3 (EU PIC Interreg IIIB 2000-2006, MAC/4.2/M12, 03/MAC/4.2/M8, 05/MAC/4.2/M11), REDECA (Spanish Government, CTM2005-07712-C03/MAR) and MARPROF (EU PCT MAC 2007-2013, MAC/2/M065).

\section{REFERENCES}

Abelló P., Valladares F.J., Castellón A. 1988. Analysis of the structure of decapod crustacean assemblages off the Catalan coast (North-West Mediterranean). Mar. Biol. 98: 39-49. http://dx.doi.org/10.1007/BF00392657

Afonso P., Porteiro F.M., Fontes J., et al. 2013. New and rare coastal fishes in the Azores islands: occasional events or tropicalization process? J. Fish Biol. 83: 272-294. http://dx.doi.org/10.1111/jfb.12162

Almaça C. 1960. Variabilidade de alguns caracteres usados na taxonomia do gen. Carcinus Leach. Revista da Faculdade de Ciências, Universidade de Lisboa, ser. 2a, C, 8(2): 137-154 + tabl. 1-20.

Araújo R., Calado R. 2003. Crustáceos Decápodes do Arquipélago da Madeira. Biodiversidade madeirense: avaliação e conservação, 4. Direcção Regional do Ambiente. Funchal, Madeira: $236 \mathrm{pp}$

Araújo R., Biscoito M., Santana J.I., et al. 2009. First record of the deep-sea red crab Chaceon inglei (Decapoda: Geryonidae) from Madeira and the Canary Islands (northeastern Atlantic Ocean). Bocagiana 230: 1-6.

Araújo R., Biscoito M., Santana J.I., et al. 2014 (in press). New records of deep-sea decapod crustaceans from off the Archipelago of Madeira (north-eastern Atlantic). Bol. Mus. Hist. Nat. Funchal, 64(339).

Barquín-Diez J., Carrillo M. 1988. Los cangrejos (Crustacea, Decapoda, Bachyura) de las Islas Canarias. In: Gállego L. (coord.), Bentos VI, Resúmenes VI Simposio Ibérico de Estudio del Bentos Marino. Ed. Bílbilis, Palma de Mallorca, $65 \mathrm{pp}$.

Barquín-Diez J., Moreno Batet E., Pérez Dionis G. 1982-1983. Crustáceos Decápodos. In: Bacallado J.J. (dir.) et al., Estudio del Bentos Marino del Archipiélago Canario. Catálogo preliminar de los invertebrados marinos bentónicos de Canarias. Confección de un manual de identificación. Gobierno de Canarias. La Laguna: I, 52-58, 84-87, 101-107, 123-124, 144-145; II, 175-176; III, 335, 466-515, 693-696, 712-717, 729-731, 754$755,782,785-788$.
Barton E.D., Arístegui J., Tett P., et al. 1998. The transition zone of the Canary Current upwelling region. Prog. Oceanogr. 41: 455-504.

http://dx.doi.org/10.1016/S0079-6611(98)00023-8

Barrois T. 1888. Catalogue des Crustacés marins recueillis aux Açores, durant les mois d'Août et Septembre 1887. Le Bigot Frères. Lille, France: 110 pp. + lám. I-IV.

Biscoito M., Freitas M., Pajuelo J.G., et al. 2015. Sex-structure, depth distribution, intermoult period and reproductive pattern of the deep-sea red crab Chaceon affinis (Brachyura, Geryonidae) in two populations in the north-eastern Atlantic. Deep-Sea Res. I 95: 99-114. http://dx.doi.org/10.1016/j.dsr.2014.10.010

Borges P.A.V., Costa A., Cunha R., et al. (eds) 2010. A list of the terrestrial and marine biota from the Azores. Princípia, Oeiras, $432 \mathrm{pp}$.

Bouvier E.L. 1922. Observations complémentaires sur les Crustacés décapodes (Abstraction faite des Carides) provenant des Campagnes de S.A.S. le Prince de Monaco. Résult. Camp. sci. Monaco 62: 1-106.

Bouvier E.L. 1940. Décapodes Marcheurs. Faune de France 37: $1-404$.

Braun J.G., Molina R. 1984. El mar. In: Varios Autores, Geografía de Canarias. 1. Geografía Física. Editorial Interinsular Canaria, Santa Cruz de Tenerife, pp. 17-28.

Brito A. 2010. Biogeografía y conservación de la biodiversidad marina en la Macaronesia. Rev. Acad. Can. Cienc. 22(3): 215-229.

Brito A., Pascual P.J., Falcón J.M., et al. 2002. Peces de las Islas Canarias. Catálogo comentado e ilustrado. F. Lemus Editor, La Laguna, $419 \mathrm{pp}$.

Brito A., Falcón J.M., Herrera R. 2005. Sobre la tropicalización reciente de la ictiofauna litoral de las islas Canarias y su relación con cambios ambientales y actividades antrópicas. Vieraea 33: 515-525.

Brito A., Falcón, J.M., Herrera R. 2007. Características zoogeográficas de la ictiofauna litoral de las Islas de Cabo Verde y comparación con los archipiélagos macaronésicos. Rev. Acad. Can. Cienc. 18(4): 93-109.

Brullé M. 1837-1839. Crustacés. In: Webb P.B., Berthelot S., Histoire naturelle des Iles Canaries, Zoologie 2 (2: Entomologie): 13-18, 1 pl. (Crustacés) Atlas (1837); Text (1839). Paris.

Castro J.J. 2012. A new record of the West African fiddler crab, Uca tangeri, from Gran Canaria (Canary Islands). Mar. Biodivers. Rec. 5: e19. http://dx.doi.org/10.1017/s1755267212000036

Chapman G., Santler J.E. 1955. Aspects of the fauna and flora of the Azores. V. Crustacea. Ann. Mag. nat. Hist. (ser. 12) 18: 371-376.

Dellinger T., Davenport J., Wirtz P. 1997. Comparisons of social structure of Columbus crabs living on loggerhead sea turtles and inanimate flotsam. J. Mar. Biol. Assoc. U. K. 77(1): 185-194. http://dx.doi.org/10.1017/S0025315400033865

Doflein F. 1904. Brachyura. Wissenschaftliche Ergebnisse der Deutschen Tiefsee-Expedition aus dem Dampfer 'Valdivia', 1898-1899, Jena, 6: i-xiv + 1-314 + pl. 1-58 (2 vols.).

D’Udekem d'Acoz C. 1999. Inventaire et distribution des crustacés décapodes de l'Atlantique nord-oriental, de la Méditerranée et des eaux continentales adjacentes au nord de $25^{\circ} \mathrm{N}$. Patrimoines naturels 40: $\mathrm{i}-\mathrm{x}+1-383$.

Dürr J., González J.A. 2001. Feeding habits of Beryx splendens and Beryx decadactylus (Berycidae) off the Canary Islands. Fish. Res. 54(3): 363-374. http://dx.doi.org/10.1016/S0165-7836(01)00269-7

Espino F., González J.A., Boyra A., et al. 2014. Diversity and biogeography of fishes in the Arinaga-Gando area, east coast of Gran Canaria (Canary Islands). Rev. Acad. Can. Cienc. 26: 9-25.

Fanlo M., Carrillo J., González J.A. 1993. Régimen alimentario de Pagrus pagrus (L.) (Osteichthyes, Sparidae) en Canarias. In: Estudios del Bentos Marino, Publ. Espec. Inst. Esp. Oceanogr. 11: 27-32.

Fanlo M., González J.A., Carrillo J. 1996. Régimen alimentario de Pagellus erythrinus (L.) (Osteichthyes, Sparidae) en las islas Canarias. In: Llinás O., González J.A., Rueda M.J. (eds), Oceanografía y Recursos Marinos en el Atlántico CentroOriental. Instituto Canario de Ciencias Marinas, Gran Canaria, pp. 271-281.

Forest J. 1964. Le genre Macropodia Leach en Méditerranée, II: Remarques sur la nomenclature et les synonymies (Crustacea Brachyura Majidae). Bull. Mus. Hist. nat. Paris 36(3): 348-354.

Forest J. 1978. Le genre Macropodia Leach dans les eaux atlan- 
tiques européennes (Crustacea Brachyura Majidae). Cah. Biol. Mar. 19: 323-342.

Forest J., Guinot D. 1966. Crustacés Décapodes: Brachyoures. In: Campagne de la Calypso dans le golfe de Guinée et aux îles Principe, Sâo Tomé et Annobon (1956), 16. Ann. Inst. océanogr. (Monaco) 44: 23-124.

Forest J., Zariquiey Álvarez R. 1964. Le genre Macropodia Leach en Méditerranée, I: Description et étude comparative des espèces (Crustacea Brachyura Majidae). Bull. Mus. Hist. nat. Paris, ser. 2, 36(2): 222-244

Fransen C.H.J.M. 1991. Preliminary report on Crustacea collected in the eastern part of the North Atlantic during the CANCAP and Mauritania expeditions of the former Rijksmuseum van Natuurlijke Historie, Leiden. Nationaal Naturhistorisch Museum, Leiden: i-vi + 1-200.

Fransen C.H.J.M. 2015a. Carcinus maenas (Linnaeus, 1758). Accessed on 2015-10-29 through: World Register of Marine Species. http://www.marinespecies.org/aphia.php? $\mathrm{p}=$ taxdetails\&id=107381

Fransen C.H.J.M. 2015b. Carcinus aestuarii Nardo, 1847. Accessed on 2015-08-05 through: World Register of Marine Species. http://marinespecies.org/aphia.php? $\mathrm{p}=$ taxdetails \&id=107380

Fransen C.H.J.M., Wirtz P. 1997. Contribution to the knowledge of decapod crustaceans from Madeira and from the Canary Islands. Zool. Meded. Leiden 71(19): 215-230.

García Cabrera C. 1971. Interrelaciones entre las faunas marinas de las Antillas y Canarias. Anuario de Estudios Atlánticos 17: 37-55.

García-Raso J.E. 1993. New record of other African species of Crustacea Decapoda, Cycloes cristata (Brullé), from European and Mediterranean waters. Bios 1(1): 215-221.

González J.A. 1995. Catálogo de los Crustáceos Decápodos de las islas Canarias. Publicaciones Turquesa, Santa Cruz de Tenerife, $282 \mathrm{pp}$.

González J.A., Quiles, J.A. 2003. Orden Decapoda. In: Moro L., Martín J.L., et al. (eds), Lista de especies marinas de Canarias (algas, hongos, plantas y animales), Gobierno de Canarias y Gesplan, La Laguna: pp. 74-80, 214-215.

González J.A., Santana J.I., Fernández-Vergaz V. 1996. The family Geryonidae (Decapoda, Brachyura) in the Canary Islands. Crustaceana 69(5): 627-635. http://dx.doi.org/10.1163/156854096X00637

González J.A., Quiles J.A., Santana J.I. 2000. The family Calappidae (Decapoda, Brachyura) around the Canary Islands. Crustaceana 73(8): 1007-1014 http://dx.doi.org/10.1163/156854000504912

González J.A., García-Mederos A.M., Pérez-Peñalvo J.A., et al. 2004. Prospección con nasas para crustáceos y peces en aguas profundas de las Islas Cabo Verde. Resultados de la campaña "Taliarte 2003-08". Inf. Téc. Inst. Canario Cienc. Mar. 11: 1-76.

González J.A., Martín L., Herrera R., et al. 2012a. Cirripedia of the Canary Islands: distribution and ecological notes. J. Mar. Biol. Assoc. U.K. 92(1): 129-141.

González J.A., Pajuelo J.G., Lorenzo J.M., et al. 2012b. Talla Mínima de Captura de peces, crustáceos y moluscos de interés pesquero en Canarias. Una propuesta científica para su conservación. In: González J.A., Pajuelo J.G., Lorenzo J.M. (eds). Gobierno de Canarias. Las Palmas de Gran Canaria, 252 pp.

González-Gurriarán E. 1987. El patexo, Polybius henslowii Leach (Decapoda Brachyura), en las costas de Galicia (NW de España): I. Distribución espacial y cambios temporales. Inv. Pesq., 51(Supl. 1): 361-374.

Guinot D. 1969. Sur divers Xanthidae notamment sur Actaea de Haan et Paractaea gen. nov. (Crustacea Decapoda Brachyura). Cah. Pacif. 13: 223-267.

Hartnoll R.G., Rice A.L., Williamson D.I. 1987. Dorhynchus thomsoni: a deep sea crab with a strange life story. Inv. Pesq. 51(Supl. 1): 125-133.

Heller C. 1863. Die Crustaceen des südlichen Europa. Crustacea Podophthalmia. Mit einer übersicht über die horizontale Verbreitung sämmtlicher europäischer Arten: xi + 336 pp.

Herrera R., Montero D., Haroun R. 1993. Bionomía bentónica del litoral de la playa del Cabrón (Gran Canaria). In: Estudios del Bentos Marino, Publ. Espec. Inst. Esp. Oceanogr. 11: 291-298.

Holthuis L.B. 1995. Prólogo. In: González J.A., Catálogo de los Crustáceos Decápodos de las Islas Canarias. Publicaciones Turquesa, Santa Cruz de Tenerife, pp. 9-10, 1 fig.

Holthuis L.B., Gottlieb E. 1958. An annotated list of Decapod Crustacea of the Mediterranean Coast of Israel, with an appendix listing the Decapoda of the Eastern Mediterranean. Bull. Res. Counc. Israel 7B(1-2): 1-126.
Horta Costa B., Assis J., Franco G., et al. 2014. Tropicalization of fish assemblages in temperate biogeographic transition zones. Mar. Ecol. Prog. Ser. 504: 241-252. http://dx.doi.org/10.3354/meps 10749

Landeira J.M. Lozano-Soldevilla F Hernández-León S et al. 2009. Horizontal distribution of invertebrate larvae around the oceanic island of Gran Canaria: the effect of mesoscale variability. Sci. Mar. 73: 757-767. http://dx.doi.org/10.3989/scimar.2009.73n4757

Landeira J.M., Lozano-Soldevilla F., Hernández-León S., et al. 2010. Spatial variability of planktonic invertebrate larvae in the Canary Islands area. J. Mar. Biol. Assoc. U.K. 90: 1217-1225. http://dx.doi.org/10.1017/S0025315409990750

Manning R.B. 1993. West African pinnotherid crabs, subfamily Pinnotherinae (Crustacea, Decapoda, Brachyura). Bull. Mus. natl. Hist. Nat., Paris, ser. 4, 15A (1-4): 125-177.

Manning R.B., Holthuis L.B. 1981. West African Brachyuran Crabs (Crustacea: Decapoda). Smithson. Contr. Zool. 306: i-xii + 1-379.

Marco-Herrero E., Torres A.P., Cuesta J.A., et al. 2013. The systematic position of Ergasticus (Decapoda, Brachyura) and allied genera, a molecular and morphological approach. Zool. Scr. 42(4): 427-439. http://dx.doi.org/10.1111/zsc. 12012

Marco-Herrero E., Abelló P., Drake P., et al. 2015. Annotated checklist of brachyuran crabs (Crustacea: Decapoda) of the Iberian Peninsula (SW Europe). Sci. Mar. 79(2): 243-256. http://dx.doi.org/10.3989/scimar.04161.27a

Miers E.J. 1881. On a Collection of Crustacea made by Baron Hermann Maltzam at Goree Island, Senegambia. Ann. Mag. nat. Hist., ser. 5, 8: 204-220, 259-281, 364-377.

Milne-Edwards A. 1869. Description de quelques espèces nouvelles de Crustacés provenant du voyage de M. A. Bouvier aux Iles du Cap Vert. Rev. Mag. Zool. 21: 350-355, 374-378, 409-412 [pp.1-13 on separate].

Milne-Edwards A., Bouvier E.L. 1894. Brachyures et Anomoures. In: Crustacés décapodes provenant des campagnes du yacht l'Hirondelle (1886, 1887, 1888), Première Partie. Résult. Camp. sci. Monaco 7: 1-112.

Milne-Edwards A., Bouvier E.L. 1900. Brachyures et Anomoures. In: Crustacés Décapodes, Première Partie. Expéd. sci. Travailleur et Talisman 6: 1-396. Paris.

Monod T. 1956. Hippidea et Brachyura ouest-africains. Mém. I.F.A.N. 45: 1-674.

Moreno-López A., Tuset V.M., González J.A., et al. 2002. Feeding habits of Serranus scriba (Osteichthyes, Serranidae) in the Marine Reserve of Lanzarote (Canary Islands). Bol. Mus. Mun. Funchal (Hist. Nat.) 53(302): 5-17.

Moro L., Herrera R., Ortea J., et al. 2014. Aportaciones al conocimiento y distribución de los decápodos y estomatópodos (Crustacea: Malacostraca) de las islas Canarias. Rev. Acad. Canar. Cienc. 26: 33-82.

Neumann V. 1996. On the status of Maja brachydactyla (Balss, 1922), a spider crab closely related to Maja squinado (Herbst, 1788) (Decapoda: Brachyura: Majidae). In: 6th Colloquium Crustacea Decapoda Mediterranea, Florence 12-15 Sep. 1996, Abstract, Museum of Zoology of the University of Florence, "La Specola": 64.

Ng P.K., Guinot D., Davie P.J. 2008. Systema brachyurorum: Part I. An annotated checklist of extant brachyuran crabs of the world. Raff. Bull. Zool. 17: 1-286.

Núñez J., Núñez L., Maggio Y. 2011. Invertebrados que se comercializan en Canarias como cebo vivo para la pesca deportiva. GESPLAN, Gobierno de Canarias, La Laguna, Tenerife.

Occhipinti-Ambrogi A. 2007. Global change and marine communities: Alien species and climate change. Mar. Pollut. Bull. 55: 342-352. http://dx.doi.org/10.1016/j.marpolbul.2006.11.014

Pajuelo J.G., Triay-Portella R., Santana J.I., et al. 2015. The community of deep-sea decapod crustaceans between 175 and 2600 $\mathrm{m}$ in submarine canyons of a volcanic oceanic island (centraleastern Atlantic). Deep-Sea Res. I, 105: 83-95. http://dx.doi.org/10.1016/j.dsr.2015.08.013

Perry A.L., Low P.J., Ellis J.R., et al. 2005. Climate change and distribution shifts in marine fishes. Science 308: 1912-1915. http://dx.doi.org/10.1126/science.1111322

Quiles J.A., Santana J.I., González J.A. 2002. The family Leucosiidae (Decapoda, Brachyura) around the Canary Islands. Crustaceana 74(1): 1267-1278

Reuschel S., Schubart C.D. 2006. Phylogeny and geographic differ- 
entiation of Atlanto-Mediterranean species of the genus Xantho (Crustacea: Brachyura: Xanthidae) based on genetic and morphometric analyses. Mar. Biol. 148(4): 853-866. http://dx.doi.org/10.1007/s00227-005-0095-1

Santaella E. 1973. Estudio de los Crustáceos Decápodos (excepto Peneidea) del archipiélago canario, con especial referencia a las especies de la Sección Brachyura. Ph.D. thesis, Universidad de La Laguna, 654 pp.

Santaella E. 1974. Crustáceos decápodos del Archipiélago Canario. I. Contribución al conocimiento de las especies de braquiuros (Crustacea, Decapoda, Brachyura). Bol. Inst. Esp. Oceanogr. 182: $1-31$.

Schubart C.D., Reuschel S. 2009. A proposal for a new classification of Portunoidea and Cancroidea (Brachyura: Heterotremata) based on two independent molecular phylogenies. In: Martin J.W., Crandall K.A., Felder D.L. (eds), Decapod Crustacean Phylogenetics. Crustac. Issues 18: 533-549.

Spalding M.D., Fox H.E., Allen G.R., et al. 2007. Marine ecoregions of the world: A bioregionalization of coastal and shelf areas. BioScience 57(7): 573-583. http://dx.doi.org/10.1641/B570707

Spiridonov V.A., Neretina T.V., Schepetov D. 2014. Morphological characterization and molecular phylogeny of Portunoidea Rafinesque, 1815 (Crustacea Brachyura): Implications for understanding evolution of swimming capacity and revision of the family-level classification. Zool. Anz. 253(5): 404-429. http://dx.doi.org/10.1016/j.jcz.2014.03.003

Triay-Portella R., González J.A., Santana J.I., et al. 2014. Reproductive pattern and egg development of the deep-sea crab Paromola cuvieri (Brachyura, Homolidae) around the Canary Islands (NE Atlantic). Deep-Sea Res. I 85: 1-14. http://dx.doi.org/10.1016/j.dsr.2013.12.001

Triay-Portella R., Pajuelo J.G., Manent P., et al. 2015. New records of non-indigenous fishes (Perciformes and Tetraodontiformes) from the Canary Islands (eastern Atlantic). Cybium 39(3): 163-174.
Türkay M. 1976. Die Madeirensischen Brachyuren des Museu Municipal do Funchal und des Forschungs Instituts Senckenberg, I: Familien Dromidae, Homolidae, Calappidae, Leucosiidae, Cancridae, Portunidae, Xanthidae, Geryonidae, Goneplacidae und Palicidae (Crustacea: Decapoda). Bol. Mus. Mun. Funchal 30(133): 57-74

Türkay M. 1982. Marine Crustacea Decapoda von den Kapverdischen Inseln mit Bemerkungen zur Zoogeographie des Gebietes. Cour. Forsch. Inst. Senckenberg 52: 91-129.

Türkay M. 2001. Decapoda. In: Costello M.J., Emblow C., White R. (eds), European register of marine species. A check-list of the marine species in Europe and a bibliography of guides to their identification. Patrimoines Naturels 50: 284-292.

Tuset V.M., González J.A., García Díaz M.M., et al. 1996. Feeding habits of Serranus cabrilla (Serranidae) in the Canary Islands. Cybium 20(2): 161-167.

Vergés A., Steinberg P.D., Hay M.E., et al. 2014. The tropicalization of temperate marine ecosystems: climate-mediated changes in herbivory and community phase shifts. Proc. R. Soc. B 281 (1789): $1-10$ http://dx.doi.org/10.1098/rspb.2014.0846

Wirtz P., d'Udekem d'Acoz C. 2001. Decapoda from Antipatharia, Gorgonaria and Bivalvia at the Cape Verde Islands. Helgol. Mar. Res. 55: 112-115. http://dx.doi.org/10.1007/s101520100073

Wirtz P., Martins H.R. 1993. Notes on some rare and little known marine invertebrates from the Azores, with a discussion of the zoogeography of the region. Arquipélago - Life Mar. Sci. 11A: 55-63.

Wirtz P. Fricke R., Biscoito M J. 2008. The coastal fishes of Madeira Island - new records and an annotated check-list. Zootaxa 1715: 1-26.

Wirtz P., Brito A., Falcón J.M., et al. 2013. The coastal fishes of the Cape Verde Islands - new records and an annotated check-list. Spixiana 36(1): 113-142.

Zariquiey Álvarez R. 1968. Crustáceos decápodos ibéricos. Inv. Pesq., 32: i-Xv + 1-510. 